\title{
Association of Lymphatic Abnormalities with Early Complications after Fontan Operation
}

\author{
Sven Dittrich ${ }^{10}$ Anja Weise ${ }^{1}$ Robert Cesnjevar ${ }^{2}$ \\ Julia Moosmann $^{1}$ () Martin Glöckler $^{1}$ \\ ${ }^{1}$ Department of Pediatric Cardiology, Friedrich-Alexander-Universitat \\ Erlangen-Nürnberg, Erlangen, Germany \\ 2 Department of Pediatric Cardiac Surgery, University Hospital \\ Erlangen, Erlangen, Bavaria, Germany \\ 3 Radiology Institute, Friedrich-Alexander-Universitat Erlangen- \\ Nürnberg, Erlangen, Germany \\ ${ }^{4}$ Department of Pediatric Cardiac Surgery, Friedrich-Alexander- \\ Universitat Erlangen-Nürnberg, Erlangen, Germany
}

Oliver Rompel ${ }^{3}$ André Rüffer ${ }^{4}$ Martin Schöber ${ }^{1}$

Address for correspondence Sven Dittrich, PhD, MD, Department of Pediatric Cardiology, Friedrich-Alexander-Universitat ErlangenNürnberg, Loschgestrasse 15, Erlangen 91054, Germany (e-mail: Sven.Dittrich@uk-erlangen.de).

Thorac Cardiovasc Surg 2021;69:e1-e9.

\section{Abstract \\ Keywords \\ - congenital heart disease \\ - Fontan operation \\ - lymphatic perfusion pattern \\ - cardiovascular magnetic resonance imaging}

Background Increased central venous pressure is inherent in Fontan circulation but not strongly related to Fontan complication. Abnormalities of the lymphatic circulation may play a crucial role in early Fontan complications.

Methods This was a retrospective, single-center study of patients undergoing Fontan operation from 2008 to 2015. The primary outcome was significant early Fontan complication defined as secondary in-hospital treatment due to peripheral edema, ascites, pleural effusions, protein-losing enteropathy, or plastic bronchitis. All patients received T2-weighted magnetic resonance images to assess abdominal and thoracic lymphatic perfusion pattern 6 months after Fontan completion with respect to localization, distribution, and extension of lymphatic perfusion pattern (type 1-4) and with application of an area score ( $0-12$ points).

Results Nine out of 42 patients developed early Fontan complication. Patients with complication had longer chest tube drainage (mean 28 [interquartile range [IQR]: 13-60] vs. 13 [IQR: 2-22] days, $p=0.01$ ) and more often obstructions in the Fontan circuit 6 months after surgery ( 56 vs. $15 \%, p=0.02$ ). Twelve patients showed little or no abnormalities of lymphatic perfusion (lymphatic perfusion pattern type 1). Most frequently magnetic resonance imaging showed lymphatic congestion in the supraclavicular region (24/42 patients). Paramesenteric lymphatic congestion was observed in eight patients. Patients with early Fontan complications presented with higher lymphatic area score (6 [min-max: $2-10$ ] vs. 2 [min-max: $0-8]$ ), $p=0.001)$ and greater distribution and extension of thoracic lymphatic congestion (type $3-4: n=5 / 9$ vs. $n=1 / 33, p=0.001$ ).

Conclusion Early Fontan complication is related to hemodynamic factors such as circuit obstruction and to the occurrence and extent of lymphatic congestion. received

July 9,2020

accepted after revision

October 30, 2020

published online

December 31, 2020
DOI https://doi.org/

10.1055/s-0040-1722178. ISSN 0171-6425.
(C) 2020. The Author(s).

This is an open access article published by Thieme under the terms of the Creative Commons Attribution-NonDerivative-NonCommercial-License, permitting copying and reproduction so long as the original work is given appropriate credit. Contents may not be used for commercial purposes, or adapted, remixed, transformed or built upon. (https://creativecommons.org/ licenses/by-nc-nd/4.0/)

Georg Thieme Verlag KG, Rüdigerstraße 14, 70469 Stuttgart, Germany 


\section{Introduction}

Undeniable progress in the treatment and care of congenital univentricular heart defects has been made over the past years. However, patients after Fontan operation continue to experience a significantly increased risk of morbidity and mortality. ${ }^{1,2}$ Protein-losing enteropathy (PLE) is a rare and serious complication of the Fontan circulation with an incidence of 3 to $14 \%^{2,3}$ Symptoms of PLE include edema, hypoproteinemia, low serum albumin and dysentery, which often aggravate over time and clinically appear in an undulating course. In a previous multicenter study PLE patients showed edema and ascites in more than $50 \%$, pleural effusions in $20 \%$, and dysentery in $10 \%$ at the time of diagnosis. ${ }^{3}$ The time between Fontan completion and the clinical onset of PLE symptoms is very variable ranging from months to decades. Earlier studies report mean intervals between surgery and PLE diagnosis of 2.7 to 13 years. ${ }^{2-4}$ Limitations and disturbances of the Fontan circuit are well described ${ }^{5}$ including restricted ventricular function, low cardiac output, venous congestion, and increased mesenteric vascular resistance, which might contribute to PLE development. ${ }^{2,6}$ On the other hand, PLE also occurs in "good" Fontan patients with low pulmonary vascular resistance and low central venous pressure. $^{4,7}$ PLE is a severe end-organ dysfunction. ${ }^{8}$ The increased intestinal loss of proteins leads to low serum protein levels and peripheral edema, hypercoagulability, and immunodeficiency due to intestinal loss of lymphocytes and low serum immunoglobulin levels. ${ }^{2,9,10}$ Five-year mortality of PLE has been reported up to $50 \%$ in the past ${ }^{3,11}$ and improved to 6 to $12 \%$ in more recent studies. ${ }^{2,12}$ Independent or related to the causes of PLE intestinal inflammation might contribute to the disease development ${ }^{6}$ and suppressing intestinal inflammation seems to improve prognosis. ${ }^{2}$

However, the question is when and how does PLE begin in patients with univentricular heart defects? As mentioned above, the development of the full clinical picture of PLE takes months to years. ${ }^{2-4}$ Prior to the clinical onset of PLE postoperative problems such as peripheral edema, ascites or prolonged drainage of pleural effusions are often already apparent in the clinical course after Fontan completion with an incidence of up to $24 \%{ }^{13}$ and have been reported as "early" Fontan complications. ${ }^{14}$ Preoperative lymphatic abnormalities in the neck and thorax have been recently reported to be associated with early Fontan complications. ${ }^{13,15}$ In addition, a growing number of recent studies suggest that lymphatic congestion is an independent factor for the development of PLE $^{13,15}$ or plastic bronchitis. ${ }^{13,16}$ However, it is not shown that early Fontan complications precede later PLE manifestation and therefore it is still speculative to assume a possible relevance of abnormal lymphatic perfusion pattern after upper cavopulmonary connection and after total cavopulmonary connection on the onset of PLE.

The focus of this study is to investigate the association of early Fontan complications to thoracic and abdominal lymphatic perfusion pattern assessed with T2-weighted magnetic resonance imaging (MRI) 6 months after Fontan completion.

\section{Patients and Methods}

\section{Study Inclusion and Exclusion Criteria}

Our in-house hospital database was reviewed to identify patients who have received primary Fontan completion at preschool age at the Department of Pediatric Cardiac Surgery at the Friedrich-Alexander University Erlangen-Nürnberg between September 2007 and August 2015. As part of our follow-up routine protocol, all patients are scheduled for cardiac MRI scan 6 months after surgery if no contraindications are present. All patients after Fontan completion received oral anticoagulation with phenprocoumon that was changed to low-dose aspirin if the 6-month MRI scan has confirmed absence of thrombosis, none-obstructive Fontan circulation, adequate ventricular function, adequate atrioventricular (AV), and aortic valve function, and adequate bilateral pulmonary artery perfusion. Since we first detected thoracic lymphatic congestion in Fontan patients in 2006, ${ }^{17}$ additional T2weighted sequences of thorax and abdomen were included in our routine MRI scan protocol for clinical and scientific purpose. The local ethics committee (Ref.-No. 3738) approved this approach and the data analysis for the study. The only exclusion criteria for data analysis in this study were missing T2-weighted MRIs 6 months after Fontan completion or a preceding Kawashima operation in patients with hemiazygos-vein drainage and absence of the inferior vena cava.

The primary outcome was significant early Fontan complication defined as secondary in-hospital treatment due to peripheral edema, ascites, pleural effusions, PLE, or plastic bronchitis within 6 months follow-up after surgery. Primary outcome was analyzed concerning typical pre- and perioperative conditions and lymphatic imaging in the 6-month postoperative MRI scan.

\section{Data Collection}

A retrospective medical record review was performed for all patients. Demographic data included sex, age, and weight at Fontan operation. Body surface area (BSA) was calculated according to the modified formula of DuBois and DuBois: $\mathrm{BSA}=\left(W^{0.425} \times H^{0.725}\right) \times 0.007184[W=$ weight in kilograms, $H=$ height in centimeters]. ${ }^{18}$ Clinical variables included main diagnosis of the cardiac defect, dominance of left or right ventricle, surgical history, catheterization history (including mean blood pressure of left and right pulmonary artery and systemic ventricular pressures), and echocardiography assessment of possible AV valve regurgitation. Surgical variables included conduit size, the use of fenestration during the Fontan procedure, and cardiopulmonary bypass time. Postoperative clinical variables included total time requiring chest tube drainage (from the day of surgery until removal of the last drainage before discharge), time on mechanical ventilation, and central venous pressure 1 hour after extubation. Sixmonth MRI included analysis of ventricular function, AV-valve function, vessel or anastomoses obstruction, and diameter of vessels to calculate McGoon ratio and Nakata index. ${ }^{19,20}$ Vessel or anastomoses obstruction were defined as $>20 \%$ reduction in the prestenotic diameter. In addition, pulmonary artery obstruction was diagnosed if a perfusion mismatch between 
the left and the right pulmonary artery was $>2$ : 1 . Early Fontan complication was defined as mentioned above. The reasons for complications were classified according to the Book categorization $^{14}$ and the modifications suggested by Ghosh et $\mathrm{al}^{13}$ : group 1 (any obstruction at any location in the Fontan circuit), group 2 (at least moderate systolic or diastolic dysfunction and/or at least moderate AV-valve regurgitation), group 3 (pleural effusions in the absence of group 1 or group 2 conditions), and group 4 (lymphatic failure manifesting as chylothorax or plastic bronchitis). The severity and long-term effects of early Fontan complications were not analyzed in this cross-sectional study, which was completed with the 6-month postoperative MRI scan. The worst possible outcome of Fontan completion, failure of Fontan circulation, was defined as death, the need for Fontan takedown or listing for heart transplantation.

\section{Assessment of Lymphatic Perfusion Pattern}

T2-weighted images were used to characterize lymphatic perfusion pattern. After preview-visualization of all T2 images of all patients, we defined five regions with possible signal enhancement: thoracic supraclavicular region (left/right), the axillary region (left/right), and abdominal paramesenteric region. We used sections in frontal reconstruction and evaluated them visualized in Maximum Intensity Projection (MIP) with a thickness of $10 \mathrm{~mm}$. In the described regions, always the position with the highest signal enhancement was selected. An area of $<$ or $\geq 3 \mathrm{~cm}^{2}$ was chosen arbitrarily as semiquantitative score to distinguish between severe and mild forms of lymphatic congestion. We hypothesized that the number and distribution of localizations with signal enhancement as well as the dimension of the affected areas might have significance on early Fontan complication. The presence and extent of lymphatic signaling were assessed separately for each region. The thoracic supraclavicular and the axillary region was scored separately for both sides (right and left): 0 (no abnormality), 1 (moderate abnormality with area of enhancement $<3 \mathrm{~cm}^{2}$ ), or 2 (extended abnormality with area of enhancement $\geq 3 \mathrm{~cm}^{2}$ ) ( $\mathbf{- F i g . 1}$ ). The paramesenteric region (no distribution into left or right) was scored with double values: 0 (no abnormality), 2 (moderate abnormality with area of enhancement $<3 \mathrm{~cm}^{2}$ ), or 4 (extended abnormality with area of enhancement $\geq 3 \mathrm{~cm}^{2}$ ) ( - Fig. 2). All numbers of thoracic and abdominal regions were added to a score ranging from 0 to 12 .

For comparability purposes, thoracic lymphatic perfusion pattern was additionally graded on the basis of recently published categorization by Biko et $\mathrm{al}^{15}$ and by Ghosh et $\mathrm{al}^{13}$ : type 1 (little or no abnormalities), type 2 (abnormal perfusion pattern in the supraclavicular region), type 3 (abnormal perfusion pattern extending into the mediastinum), type 4 (abnormal perfusion pattern extending into the lung).

\section{MRI Protocol 6 Months after Fontan Completion}

During MRI examinations, all patients received general anesthesia with laryngeal intubation or laryngeal mask ventilation and patients were imaged in supine position. While mechanical ventilation and supine position might adversely bias the Fontan hemodynamic and flow analyses, we do not expect any impact on the postoperative anatomy or lymphatic abnormalities. MRI scans were performed on a 1.5 Tesla MR scanner equipped with high-performance gradients (Magnetom Aera, Siemens AG, Erlangen, Germany). The imaging protocol included balanced steady-state free precession cine sequences for functional and volumetric analysis of both ventricles. Phase-contrast MRI was acquired for the quantification of the pulmonary to systemic

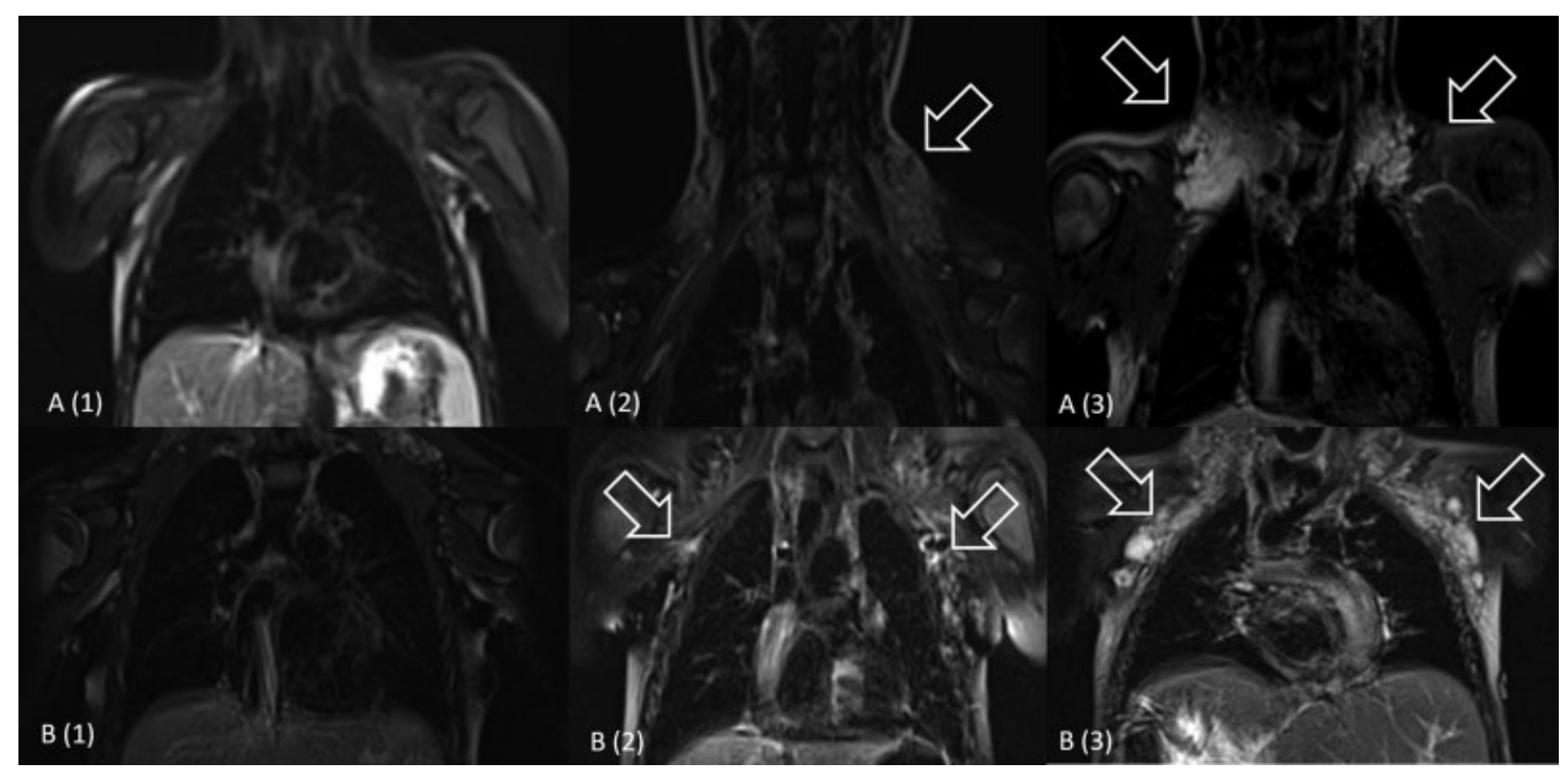

Fig. 1 Scores for lymphatic perfusion pattern in the thoracic regions. Differences of lymphatic T2-signaling in the thoracic regions: (A) upper row, supraclavicular region; (A1) no signaling right/left (score: 0/0); (A2) low signaling right < left (arrow) (score: 0/1); (A3) distinct lymphatic signaling right/left [arrows] (score: 2/2). (B) lower row, axillary region: (B1): no signaling right/left (score: 0/0); (B2) low signaling right/left with extension $<3 \mathrm{~cm}^{2}$ (arrows) (score: 1/1); (B3) distinct lymphatic signaling right > left (arrows) (score: 2/2). 

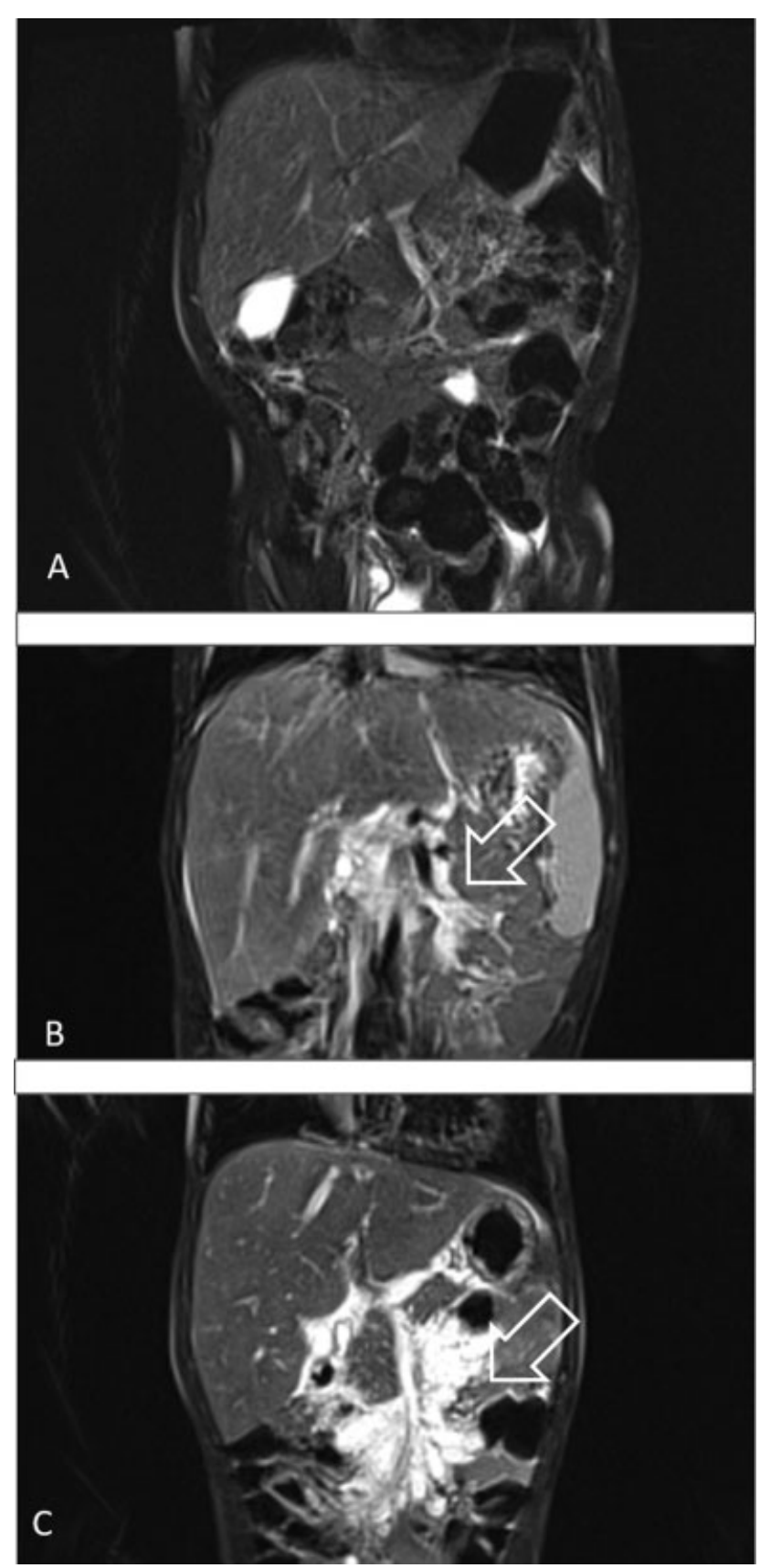

Fig. 2 Scores for lymphatic perfusion pattern in the mesenteric region. Differences of lymphatic T2-signaling in the mesenteric region: (A) no signaling (score: 0 ); (B) positive signaling, expansion $<3 \mathrm{~cm}^{2}$ (arrow) (score: 2 ); (C) positive signaling, expansion $>3 \mathrm{~cm}^{2}$ (arrow) (score: 4).

blood flow ratio (Qp:Qs), valvular regurgitation fractions and the flow velocity through valvular or vascular stenosis. Contrast-enhanced MR angiography was conducted for threedimensional depiction of the morphology of the ventricle, the pulmonary arteries, and the aortic arch. Scan parameters were as follows for all patients: slice thickness $8 \mathrm{~mm}$, in-plane resolution $2.5 \times 1.8 \mathrm{~mm}$, time to echo $1.1 \mathrm{~ms}$, time to repetition $42 \mathrm{~ms}$, and flip angle 50 degree. $^{21}$ Additionally, a T2-weighted MRI lymphatic study was performed using a respiratory navigated and cardiac-gated three-dimensional turbo spin-echo sequence with the following parameters: $256 \times 256$; field of view, 300 to 450, repetition time/echo time 2,500/650; flip angle, 140 degrees; voxel size, $1.1 \times 1.1 \times 1.1 \mathrm{~mm}$. The generated three-dimensional volume was formatted into a coronal maximal-intensity projection reconstruction of the source images.

\section{MRI Analysis}

Image data analysis was performed using commercially available software that enables post-processing of cardiac MR data (syngo.via, Siemens AG, Erlangen, Germany). Results of end-diastolic volume, end-systolic volume, stroke volume, myocardial mass, and ejection fraction (EF) were documented. End-diastolic and end-systolic volumes were calculated by summing the volume of the ventricular blood pool in each section. EF was calculated based on end-diastolic and end-systolic volumes as [EF $=$ (end-diastolic volume - endsystolic volume)/end-diastolic volume] $\times 100(\%)$. T2-images were analyzed with the same software.

\section{Statistical Analysis}

Demographic, clinical, surgical, and postoperative and outcome variables are presented using descriptive statistics. Median with interquartile range or minimum and maximum values are reported for non-normal distributed continuous variables and counts with percentages are reported for categorical variables. Nonparametric Mann-Whitney-U test was used for bivariate associations. Fisher's exact tests was used for categorical variables, with a 2 -tailed $p$-value $<0.05$. Associations between demographic or clinical and outcome variables were evaluated with Pearson's chi-squared test. All statistical analyses were performed by using IBM SPSS statistics v24.0 (Armonk, New York, United States).

\section{Results}

Between September 2007 and August 2015, 49 patients received successful primary Fontan completion at preschool age at our institution. Forty-four patients received a 6 months postoperative MRI scan and 42 patients could be included into the study analysis (one patient was excluded after Kawashima operation and in one patient T2-weighted images were missing). Time on ventilator in the intensive care unit (ICU) was 5 hours (IQR: $3.8-16.0$ hours), time with chest tube drainage was 14 days (IQR: 9-27 days) and time to discharge was 23 days (IQR: 18-32 days). Two patients showed specific complications, which prolonged the ICU stay: one patient suffered from phrenicus palsy and one patient received venovenous extracorporeal membrane oxygenation due to large intrapulmonary shunts. After management of the acute complications, both patients were discharged home and did not need secondary inhospital treatment for Fontan complications. Nine out of 42 patients $(=21 \%)$ showed early Fontan complication according to our definition, receiving secondary in-hospital treatment within a 6 months period. They presented with the following symptoms or combinations of thereof: peripheral edema in seven, ascites in five, hypoproteinemia in four, and pleural effusions in six patients. None of the nine patients developed the full clinical picture of PLE up to 6 months after surgery and 
Table 1 Baseline characteristics, perioperative, and MRI data in patients with and without early Fontan complications

\begin{tabular}{|c|c|c|c|}
\hline & $\begin{array}{l}\text { Patients with secondary in } \\
\text { hospital treatment }(n=9)\end{array}$ & $\begin{array}{l}\text { Patients without secondary in } \\
\text { hospital treatment }(n=33)\end{array}$ & p-Value \\
\hline \multicolumn{4}{|l|}{ Demographic data } \\
\hline Male sex & $n=5(56 \%)$ & $n=23(70 \%)$ & 0.45 \\
\hline Age at TCPC $(\mathrm{m})$ & 40 (IQR: 26.5-51.5) & 36 (IQR: 31.5-38.5) & 0.16 \\
\hline Weight at TCPC $(\mathrm{kg})$ & 13.8 (IQR: 12.5-14.6) & 15.4 (IQR: 14-17) & 0.13 \\
\hline Body surface area $\left(\mathrm{m}^{2}\right)$ & 0.60 (IQR: $0.58-0.62$ ) & 0.63 (IQR: $0.61-0.68$ ) & 0.6 \\
\hline \multicolumn{4}{|l|}{ Clinical variables } \\
\hline Dominance of right ventricle & $n=4(44 \%)$ & $n=12(36 \%)$ & 0.71 \\
\hline Mean blood pressure LPA $(\mathrm{mm} \mathrm{Hg})$ & 12 (IQR: 10-15) & 10 (IQR: 8-12) & 0.07 \\
\hline Mean blood pressure RPA $(\mathrm{mm} \mathrm{Hg})$ & 11 (IQR: 6.75-16.75) & 10 (IQR: 7.5-12) & 0.20 \\
\hline End diastolic ventricular pressure $(\mathrm{mm} \mathrm{Hg})$ & 9 (IQR: 7.5-11) & 10 (IQR: 9-15.5) & 0.36 \\
\hline \multicolumn{4}{|l|}{ Surgical variables } \\
\hline Conduit size $(\mathrm{mm})$ & 18 (IQR: 17-20) & 20 (IQR: 18-20) & 0.04 \\
\hline Presence of fenestration & $n=4(44 \%)$ & $n=4(12 \%)$ & 0.05 \\
\hline Cardiopulmonary bypass time (min) & 116 (IQR: 98-127) & 98 (IQR: 80-138) & 0.83 \\
\hline \multicolumn{4}{|l|}{ Postoperative variables } \\
\hline Time with chest tube $(\mathrm{d})$ & 28 (IQR: 13-60) & 13 (IQR: 8-22) & 0.01 \\
\hline Time on mechanical ventilation (h) & 5 (IQR: 3.5-18.5) & 5 (IQR: 3.5-16) & 0.71 \\
\hline $\begin{array}{l}\text { Central venous pressure } 1 \text { hour } \\
\text { after extubation }(\mathrm{mm} \mathrm{Hg})\end{array}$ & 15 (IQR: 14.5-20) & 16 (IQR: 14-20) & 0.83 \\
\hline \multicolumn{4}{|l|}{ MRI variables } \\
\hline End-diastolic volume $\left(\mathrm{mL} / \mathrm{m}^{2} \mathrm{BSA}\right)$ & 90 (IQR: 70-128) & 88 (IQR: 73-120) & 0.94 \\
\hline Ventricular EF (\%) & 47 (IQR: 38-57) & 46 (IQR: 38-54) & 0.86 \\
\hline Relevant AV insufficiency & $n=3(33 \%)$ & $n=3(9 \%)$ & 0.1 \\
\hline Fontan obstruction & $n=5(56 \%)$ & $n=5(15 \%)$ & 0.02 \\
\hline McGoon ratio & 1.9 (IQR: $1.7-2.3$ ) & 2.0 (IQR: $1.8-2.2$ ) & 0.91 \\
\hline Nakata-Index $\left(\mathrm{mm} / \mathrm{m}^{2}\right)$ & 166 (IQR: 158-299) & 221 (IQR: 166-263) & 0.96 \\
\hline
\end{tabular}

Abbreviations: AV, atrioventricular; BSA, body surface area; EF, ejection fraction; IQR, interquartile range; LPA, left pulmonary artery; MRI, magnetic resonance imaging; RPA, right pulmonary artery; TCPC, total cavopulmonary connection.

Counts for categorical variables are reported as absolute value and percentage (\%). Continuous variables are reported as median value with IQR.

none of the patients showed plastic bronchitis. Baseline characteristics did not differ in patients with or without early Fontan complications after discharge ( - Table 1). MRI 6 months after Fontan operation did not show differences of ventricular function (EF mean 47 [IQR: 38-57\%] vs. 46 [IQR: 38-54\%], $p=0.8$ ), patients with compared to patients without early Fontan complications, respectively. Early complications in the nine patients were associated with any obstruction on the Fontan circuit in five (thereof 1 with additional AV valve regurgitation), with ventricular dysfunction and/or AV valve regurgitation without any obstruction in the Fontan circuit in two and with chylothorax in two patients (-Table 2). All patients with early Fontan complications underwent transcatheter and/or medical interventions. One of the two patients classified in the group 2 complication ( - Table 2 ) suffered from severe systolic dysfunction. He developed criteria for failing Fontan and was listed for heart transplantation.
Single-variable analysis identified smaller conduit size (mean 18 [IQR: 17-20] vs. 20 [IQR: 18-20] mm, $p=0.04$ ), longer time with chest tube drainage (mean 28 [IQR: 13-60] vs. 13 [IQR: $8-22$ ] days, $p=0.01$ ), longer in-hospital stay (mean 31 [IQR: 23-45] vs. 22 [IQR: 16.5-28] days, $p=0.04$ ), and more often the presence of any obstruction in the Fontan circuit 6 months after surgery (56 vs. $15 \%, p=0.02$ ) in patients with early Fontan complications (-Table 1). The number of patients, who received a conduit fenestration, was 4 out of 9 patients with early Fontan complications and 4 out of 33 patients without. This difference did not reach statistical significance ( 44 vs. $12 \%, p=0.05$, - Table 1 ).

With regard to the presence, degree, and distribution of lymphatic perfusion pattern in the T2-weighted MRI, 12 out of 42 patients $(=29 \%)$ had no or only minor abnormalities of thoracic lymphatic perfusion pattern (-Table 4). Most frequently, MRI showed lymphatic congestion in the 
Table 2 Classification of early Fontan complications

\begin{tabular}{|l|l|}
\hline Type of early Fontan complication & \\
\hline $\begin{array}{l}\text { Group } 1 \text { (any obstruction at any location } \\
\text { in the Fontan circuit) }\end{array}$ & $\begin{array}{l}\text { Number of } \\
\text { patients } \\
\text { ( } \boldsymbol{n} \text { out of 9) }\end{array}$ \\
\hline $\begin{array}{l}\text { Group } 2 \text { (at least moderate systolic or } \\
\text { diastolic dysfunction and/or at least } \\
\text { moderate atrioventricular } \\
\text { valve regurgitation) }\end{array}$ & 2 \\
\hline $\begin{array}{l}\text { Group } 3 \text { (pleural effusions in the absence } \\
\text { of group 1 or group 2 conditions) }\end{array}$ & 0 \\
\hline $\begin{array}{l}\text { Group } 4 \text { (lymphatic failure manifesting } \\
\text { as chylothorax or plastic bronchitis) }\end{array}$ & 2 \\
\hline
\end{tabular}

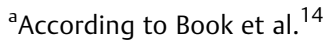

supraclavicular region (24 out of 42 patients, - Table 4). Paramesenteric lymphatic congestion was observed in eight patients (-Table 3 ).

The lymphatic area-score ranged from 2 to 10 in patients with early Fontan complication. None of the nine patients with early Fontan complication was completely free of lymphatic abnormalities (area-score $<2$, - Table 3 ). In the asymptomatic group, 8 out of 33 patients did not show any lymphatic abnormality with an area-score of " 0 ," respectively (-Table 3). Further details and absolute numbers of the regional MRI lymphatic perfusion analyses are described in -Table 4 and the differences of the relative distribution of the area-score in patients with and without early Fontan complications are visualized in - Fig. 3 . The categorization of MRI data according to the recently described four types of thoracic lymphatic perfusion pattern (type $1-4)^{13,15}$ showed that four out of nine patients with early Fontan complications were classified as type 3 or 4 . On the other hand, of the 33 patients without early complications only one patient was classified as type 3 and none as type 4 (-Table 3 ).
Patients with and without early Fontan complications showed significant differences in the thoracic and abdominal area score (median 6 [min-max: 2-10] vs. 2 [min-max: $0-8]), p=0.001$ ) and in the classification of the four types of thoracic lymphatic perfusion pattern (lymphatic perfusion pattern group $>2: n=5 / 9$ vs. $n=1 / 33, p=0.001$ ).

\section{Discussion}

The contribution of the lymphatic system to early Fontan complications and to the later development of PLE is under debate since first descriptions of thoracic lymphatic congestions in 2008. ${ }^{17,22}$ New understanding of the pathophysiological processes of the lymphatic circuit developed only after the systematic application of new lymphatic imaging techniques. ${ }^{15,16}$ Recently, Ghosh et al reported a study with 126 patients, who received T2-weighted MRI sequences to assess for lymphatic perfusion abnormalities before Fontan completion. ${ }^{13}$ This study demonstrated that patients with preoperative high-grade lymphatic abnormalities developed significant more early complications after Fontan completion. Interestingly, they reported that half of the patients developed complications related to plastic bronchitis, chylous effusions, or persistent pleural effusions in the absence of ventricular dysfunction or obstructions in the Fontan circuit. The authors concluded that these complications might be directly related to abnormal lymphatic perfusion and might be discussed as an imbalance in the physiologic interplay between lymphatic and cardiovascular circulation. ${ }^{13,23}$

Our study contributes two new aspects into the ongoing discussion: we systematically analyzed lymphatic perfusion abnormalities with T2-weighted MRI 6 months after Fontan completion and included abdominal lymphatic imaging into our analysis.

As expected, our study confirms current observations: the number of patients with early complications as defined as the need for re-admittance to hospital treatment for specific

Table 3 Thoracic and abdominal regional lymphatic area score in patients with and without early Fontan complications

\begin{tabular}{|c|c|c|c|c|}
\hline Region & Score & $\begin{array}{l}\text { Number of patients with } \\
\text { Fontan complications ( } n \text { out of } 9 \text { ) }\end{array}$ & $\begin{array}{l}\text { Number of patients without } \\
\text { Fontan complications ( } n \text { out of } 33 \text { ) }\end{array}$ & $p$-Value \\
\hline Supraclavicular right & $\begin{array}{l}0 \\
1 \\
2\end{array}$ & $\begin{array}{l}2 \\
2 \\
5\end{array}$ & $\begin{array}{l}14 \\
15 \\
4\end{array}$ & $\begin{array}{l}0.269 \\
0.208 \\
0.005\end{array}$ \\
\hline Supraclavicular left & $\begin{array}{l}0 \\
1 \\
2\end{array}$ & $\begin{array}{l}2 \\
2 \\
5\end{array}$ & $\begin{array}{l}12 \\
15 \\
6\end{array}$ & $\begin{array}{l}0.425 \\
0.208 \\
0.012\end{array}$ \\
\hline Axillary right & $\begin{array}{l}0 \\
1 \\
2\end{array}$ & $\begin{array}{l}5 \\
1 \\
3\end{array}$ & $\begin{array}{l}30 \\
2 \\
1\end{array}$ & $\begin{array}{l}0.012 \\
0.313 \\
0.006\end{array}$ \\
\hline Axillary left & $\begin{array}{l}0 \\
1 \\
2\end{array}$ & $\begin{array}{l}5 \\
1 \\
3\end{array}$ & $\begin{array}{l}27 \\
4 \\
2\end{array}$ & $\begin{array}{l}0.101 \\
0.934 \\
0.025\end{array}$ \\
\hline Paramesenteric & $\begin{array}{l}0 \\
2 \\
4\end{array}$ & $\begin{array}{l}4 \\
2 \\
3\end{array}$ & $\begin{array}{l}30 \\
2 \\
1\end{array}$ & $\begin{array}{l}0.002 \\
0.143 \\
0.006\end{array}$ \\
\hline
\end{tabular}


Table 4 Morphologic graduation of thoracic lymphatic perfusion pattern in patients with and without early Fontan complications

\begin{tabular}{|c|c|c|c|}
\hline Lymphatic perfusion pattern $^{a}$ & $\begin{array}{l}\text { Number of patients with Fontan } \\
\text { complications ( } n \text { out of } 9 \text { ) }\end{array}$ & $\begin{array}{l}\text { Number of patients without } \\
\text { Fontan complications ( } n \text { out of } 33 \text { ) }\end{array}$ & p-Value \\
\hline Type 1 (little or no abnormalities) & 1 & 11 & 0.19 \\
\hline $\begin{array}{l}\text { Type } 2 \text { (abnormal perfusion in the } \\
\text { supraclavicular region) }\end{array}$ & 3 & 21 & 0.10 \\
\hline $\begin{array}{l}\text { Type } 3 \text { (abnormal perfusion } \\
\text { extending into the mediastinum) }\end{array}$ & 2 & 1 & 0.05 \\
\hline $\begin{array}{l}\text { Type } 4 \text { (abnormal perfusion } \\
\text { extending into the lung) }\end{array}$ & 3 & 0 & 0.001 \\
\hline Type 3 or 4 (summation) & 5 & 1 & 0.001 \\
\hline
\end{tabular}

${ }^{a}$ According to Biko et al and Ghosh et al. ${ }^{13,15}$

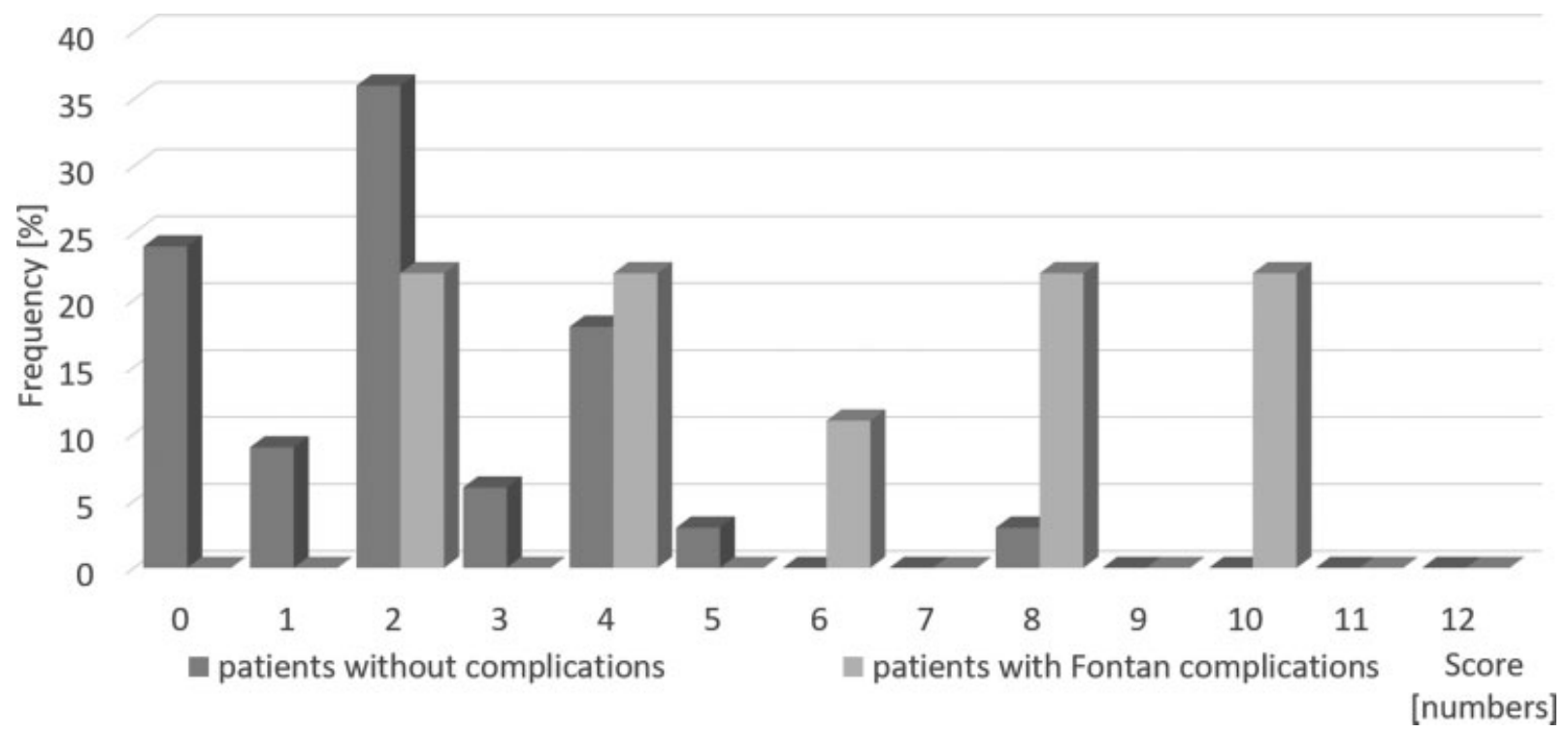

Fig. 3 Distribution of area scores of thoracic and abdominal lymphatic perfusion patterns in patients with and without Fontan complications. Median area score was 6 ( $\min -\max : 2-10$ ) versus 2 (min-max: $0-8), p=0.001$, patients with or without Fontan complications, respectively.

Fontan symptoms is relatively high (21\% in our study, $24 \%$ reported by Ghosh et $\mathrm{al}^{13}$ ).

Ghosh et al reported an association of early Fontan complications with structural failure, pump failure, prolonged effusions and lymphatic failure. ${ }^{13}$ Similarly, the majority of patients with early complications in our study showed postoperatively AV-valve or ventricular dysfunction or obstructions in the Fontan circuit, which reflects the particular need for best possible hemodynamic conditions in patients after Fontan operation. In our institute, we plan a conduit fenestration in patients, who were not considered optimal Fontan candidates from preoperative evaluation. Eight out of 42 patients in our study received a conduit fenestration and half of them developed early Fontan complications, which further underlines the importance of a well-functioning Fontan blood circuit. ${ }^{2,8}$

The rate of postoperatively thoracic lymphatic perfusion pattern abnormalities in our study is comparable to those reported by Ghosh et al before Fontan surgery. ${ }^{13}$ Ghosh et al reported normal thoracic lymphatic perfusion pattern preoperatively in $32 \%$ of patients compared with $29 \%$ postoperatively in our cohort. While a minority of $15 \%$ developed early complications in the absence of preoperative abnormalities of thoracic lymphatic perfusion pattern, ${ }^{13}$ none of our patients with normal lymphatic perfusion pattern postoperatively developed early Fontan complications. From that association, no meaningful conclusions about causality or temporal relation can be made due to the study design of our cross-sectional study. On the other hand, the association of early Fontan complications with thoracic and mesenteric lymphatic congestion rises the hypothesis of fundamental contribution of lymphatic circuit failure associated with unfavorable Fontan hemodynamics to Fontan complications. This is further supported by our observation, that early Fontan complication is associated with longer need for postoperative chest-tube drainage, which might be discussed as a malfunction of the lymphatic vessels resorbing fluid adequately from the extracellular space. ${ }^{13,23}$

What do we know today? The development of early Fontan complications and later PLE is not strictly linked to sustained unfavorable Fontan hemodynamics. ${ }^{8,13}$ Abnormalities of thoracic lymphatic perfusion pattern exist in some patients after upper cavopulmonary anastomosis and are associated with early Fontan complications. ${ }^{13}$ Development of PLE is associated 
with lymphopenia and abnormal lymphocyte subpopulation dispersion. ${ }^{9,24-26}$ Postoperative lymphatic perfusion pattern abnormalities in the 6-month MRI might be partially explained by unfavorable postoperative hemodynamics and are associated with early Fontan complications in our study. In contrast, early Fontan complications in our study did not occur in absence of pathologic lymphatic perfusion pattern.

Together, these observations may be sufficient to generate a conclusive proposal for the role of the lymphatic circulation in the development of early Fontan complications. In patients with univentricular heart disease, thoracic lymphatic drainage is impaired after surgery for upper cavopulmonary connection (Glenn operation) leading to formation of thoracic lymphatic perfusion pattern abnormalities comparable to a river delta with low slope in some patients. However, this is often not clinically manifest. ${ }^{13}$ After Fontan completion abdominal and hepatic venous pressure and blood drainage-resistance rise abruptly leading to an imbalance of increased abdominal lymphatic production and impaired thoracic lymphatic drainage. ${ }^{15}$ In some patients, this imbalance might be further affected particularly by postoperative pronounced and/or prolonged hemodynamic stress, leading to a deterioration of the mismatch of abdominal lymphatic production and thoracic drainage. Our images of paramesenteric lymphatic congestion after Fontan completion support this hypothesis as well as the fact that in our study the majority of patients with early complications suffered from unfavorable Fontan hemodynamics like vascular obstructions, ventricular dysfunction, and/or $\mathrm{AV}$ insufficiency. It is a plausible consequence that these patients had increased central venous pressure, which in turn increased lymphatic pressure resulting in lymphatic congestion. However, in some patients hemodynamic stress may improve and turn into "normal" Fontan circulation with normal pressures but persistent abnormal lymphatic perfusion pattern. If abnormal lymphatic pattern was once formed during acute hemodynamic stress, they may not dissolve in all cases and might contribute to long-lasting abnormal lymphocyte dispersion and development of PLE. ${ }^{6}$ This raises the question whether imaging of the lymphatic system should become part of routine pre- or postoperative diagnostic investigation. In the future, surgical strategies to prevent damage to the lymphatic system could attract much more attention. Further, preoperative subclinical thoracic lymphatic congestion might influence the operative strategy and the need for fenestration.

However, many questions remain unanswered. MRIs illustrate the lymphatic "highways," while the post-Fontan lymphatic microvessels especially in the liver and the bowels are largely unknown. The role of an inflammatory bowel process in the sequence of enteric protein loss, the loss of immunoglobulins, and the loss of lymphocyte remains poorly understood ${ }^{9}$ and might be an additional disease mechanism in PLE.

\section{Limitations of the Study}

We performed a cross-sectional analysis of lymphatic perfusion pattern and hemodynamics at one point 6 months after Fontan operation. For this reason, we cannot draw conclu- sions of causality and temporal relationship in the interplay of blood and lymphatic circuit. Postoperative hemodynamic imbalances, medical and vascular interventions are not considered in our analysis. Systematic analytic access to categorize lymphatic perfusion pattern with MRI techniques is rapidly evolving and our semiquantitative and arbitrary technique may have biased the results.

\section{Conclusions}

The primary outcome of this study was the prevalence and type of early Fontan complications with respect to systematic imaging of lymphatic perfusion pattern after Fontan completion. Our data confirm that early Fontan complications are partly associated with impairments of Fontan hemodynamics, and partly occur without. Even in patients without complications, smaller abnormal lymphatic perfusion pattern in the supraclavicular region is frequently found, while larger extensions of abnormal lymphatic perfusion pattern to other thoracic localizations or the mesenteric region were less frequent and associated with clinical symptoms. In contrast, normal postoperative lymphatic perfusion pattern are absent in patients with early Fontan complications. These findings rise the hypothesis of a staged development of thoracic lymphatic congestion. First, after the upper cavopulmonary connection, thoracic lymphatic vessel congestion develops especially at the regions of confluence into the subclavian veins, mostly without development of clinical symptoms. Second, after an acute rise of pressure in the lower cava vein after the Fontan completion, an imbalance between increased abdominal lymphatic production and decreased thoracic lymphatic drainage capacity develops. This may lead to an expansion of abnormal thoracic and abdominal lymphatic perfusion pattern, which is associated with early Fontan complications and which may contribute to long-term development of PLE. Therefore, we suggest that the lymphatic system should gain greater intention and lymphatic imaging should be included as a routine examination before and after Fontan completion for risk stratification.

Conflict of Interest

None declared.

\section{Acknowledgments}

The presented work was performed in fulfilment of the requirements for obtaining the degree "Dr. med" at "Friedrich-Alexander University of Erlangen-Nürnberg (FAU)" of Anja Weise.

\section{References}

1 Marshall KH, D'Udekem Y, Sholler GF, et al. Health-related quality of life in children, adolescents, and adults with a Fontan circulation: a meta-analysis. J Am Heart Assoc 2020;9(06):e014172

2 Schleiger A, Ovroutski S, Peters B, et al. Treatment strategies for protein-losing enteropathy in Fontan-palliated patients. Cardiol Young 2020;30(05):698-709

3 Mertens L, Hagler DJ, Sauer U, Somerville J, Gewillig MPle Study Group. Protein-losing enteropathy after the Fontan operation: an international multicenter study. PLE study group. J Thorac Cardiovasc Surg 1998;115(05):1063-1073 
4 Meadows J, Jenkins K. Protein-losing enteropathy: integrating a new disease paradigm into recommendations for prevention and treatment. Cardiol Young 2011;21(04):363-377

5 Gewillig M, Brown SC. The Fontan circulation after 45 years: update in physiology. Heart 2016;102(14):1081-1086

6 Rychik J. Protein-losing enteropathy after Fontan operation. Congenit Heart Dis 2007;2(05):288-300

7 Ovroutski S, Sohn C, Barikbin P, et al. Analysis of the risk factors for early failure after extracardiac Fontan operation. Ann Thorac Surg 2013;95(04):1409-1416

8 Rychik J, Atz AM, Celermajer DS, et al;American Heart Association Council on Cardiovascular Disease in the Young and Council on Cardiovascular and Stroke Nursing. Evaluation and management of the child and adult with Fontan circulation: a scientific statement from the American Heart Association. Circulation

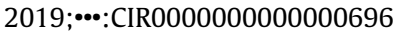

9 Alsaied T, Possner M, Brown N, et al. Lymphopenia in adults after the Fontan operation: prevalence and associations. Cardiol Young 2020;30(05):641-648

10 Udink Ten Cate FE, Hannes T, Germund I, et al. Towards a proposal for a universal diagnostic definition of protein-losing enteropathy in Fontan patients: a systematic review. Heart 2016;102(14): $1115-1119$

11 Feldt RH, Driscoll DJ, Offord KP, et al. Protein-losing enteropathy after the Fontan operation. J Thorac Cardiovasc Surg 1996;112 (03):672-680

12 John AS, Johnson JA, Khan M, Driscoll DJ, Warnes CA, Cetta F. Clinical outcomes and improved survival in patients with proteinlosing enteropathy after the Fontan operation. J Am Coll Cardiol 2014;64(01):54-62

13 Ghosh RM, Griffis HM, Glatz AC, et al. Prevalence and cause of early Fontan complications: does the lymphatic circulation play a role? J Am Heart Assoc 2020;9(07):e015318

14 Book WM, Gerardin J, Saraf A, Marie Valente A, Rodriguez F III. Clinical phenotypes of Fontan failure: implications for management. Congenit Heart Dis 2016;11(04):296-308

15 Biko DM, DeWitt AG, Pinto EM, et al. MRI evaluation of lymphatic abnormalities in the neck and thorax after Fontan surgery: relationship with outcome. Radiology 2019;291(03):774-780
16 Rychik J, Dodds KM, Goldberg D, et al. Protein losing enteropathy after Fontan operation: glimpses of clarity through the lifting fog. World J Pediatr Congenit Heart Surg 2020;11(01):92-96

17 Glöckler M, Severin T, Arnold R, et al. First description of three patients with multifocal lymphangiomatosis and protein-losing enteropathy following palliation of complex congenital heart disease with total cavo-pulmonary connection. Pediatr Cardiol 2008;29(04):771-774

18 Wang Y, Moss J, Thisted R. Predictors of body surface area. J Clin Anesth 1992;4(01):4-10

19 Gill CC, Moodie DS, McGoon DC. Staged surgical management of pulmonary atresia with diminutive pulmonary arteries. J Thorac Cardiovasc Surg 1977;73(03):436-442

20 Nakata S, Imai Y, Takanashi Y, et al. A new method for the quantitative standardization of cross-sectional areas of the pulmonary arteries in congenital heart diseases with decreased pulmonary blood flow. J Thorac Cardiovasc Surg 1984;88(04): 610-619

21 Rompel O, Janka R, May MS, et al. Cardiac MRI in children and adolescents who have undergone surgical repair of right-sided congenital heart disease: automated left ventricular volumes and function analysis and effects of different manual adjustments. RoFo Fortschr Geb Rontgenstr Nuklearmed 2015;187(12):1099-1107

22 Meadows J, Gauvreau K, Jenkins K. Lymphatic obstruction and protein-losing enteropathy in patients with congenital heart disease. Congenit Heart Dis 2008;3(04):269-276

23 Aspelund A, Robciuc MR, Karaman S, Makinen T, Alitalo K. Lymphatic system in cardiovascular medicine. Circ Res 2016; 118(03):515-530

24 Kovacikova L, Krasnanova V, Skrak P, et al. Immune abnormalities in patients with single ventricle circulation precede the Fontan procedure. World J Pediatr Congenit Heart Surg 2017;8(06): 672-682

25 Magdo HS, Stillwell TL, Greenhawt MJ, et al. Immune abnormalities in Fontan protein-losing enteropathy: a case-control study. J Pediatr 2015;167(02):331-337

26 Mattes M, Connor J, Kelly SS, Schwartz MC. Lymphopenia in patients with single-ventricle heart disease after the Fontan operation. Congenit Heart Dis 2016;11(03):270-275 